\title{
Standing Waves In Quadratic And Cubic Nonlinear Resonators: Q-Factor And Frequency Response
}

\author{
Bengt O.Enflo*, Claes M.Hedberg**, and Oleg V.Rudenko** \\ *Department of Mechanics, Kungl Tekniska Högskolan, S-1044, Sweden \\ **Blekinge Institute of Technology, S-371 79 Karlskrona, Sweden
}

\begin{abstract}
High-Q acoustic resonators are used to significantly increase the wave energy volume density. For high-intensity waves the magnitude of Q-factor is determined not only by the design of the resonator, but by the strength of internal field as well. Methods to calculate the Q-factor and both the spatio-temporal and spectral structure of this field are described. Results are given for quadratic and cubic nonlinear resonators demonstrating quite different physical properties.
\end{abstract}

Keywords: Quadratic and cubic nonlinear media, nonlinear acoustic resonator, nonlinear Qfactor.

PACS: 43.25.Gf, 43.25.Dc

\section{INTRODUCTION}

Different definitions of Q-factor are known. If one resonator wall $(x=0)$ is immovable and the other wall $(x=L)$ vibrates according to $u(x=L, t)=A \sin (\omega t)$, where $u(x, t)$ is the particle velocity, one can define $\mathrm{Q}$ as the ratio of "internal" amplitude of $u(x, t)$ and "external" amplitude $A$ of the driving force. The inequality $Q>>1$ means that inside the resonator cavity the medium vibrates much more than the boundary - the "quality" of resonator is high. As distinct from a linear system, a nonlinear generates a series of harmonics. Therefore, it is necessary to specify the meaning of "internal" amplitude of vibration. It can be the amplitude of fundamental mode, for example. However, the mean square velocity $\left\langle u(x, t)^{2}\right\rangle$ averaged over the period and over the volume of the resonant cavity seems more sophisticated. Another possible definition exploits the maximum positive peak of disturbance $u_{+}$. The Qfactor can be also the normalized maximum of the corresponding frequency response, which is the dependence of the $1^{\text {st }}$ harmonic amplitude, $\left\langle u(x, t)^{2}\right\rangle$, or $u_{+}$on the frequency shift (discrepancy) between driving frequency $\omega$ and a resonant frequency.

\section{METHOD FOR ANALYTICAL SOLUTION}

The approach based on "nonlinear superposition" of time-oscillating counterpropagating waves is used. This approach is described in detail in [1] and used by 
several authors before. For quadratic nonlinearity and harmonic vibration of one wall the functional equation is derived [1]:

$$
F\left(\omega t-k L+\frac{\varepsilon}{c} k L F\right)-F\left(\omega t+k L-\frac{\varepsilon}{c} k L F\right)=A \sin (\omega t) .
$$

The unknown function $F$ describes the velocity profile of both counter-propagating waves, $k=\omega / c$ is wave number, $\quad c$ is sound velocity and $\varepsilon$ is nonlinearity coefficient. Equation (1) can describe not only steady-state vibrations, but transient processes as well [1], including unlimited linear amplitude growth at resonance (with $\varepsilon=0$ ). If the following requirements are fulfilled - namely, the length $L$ of resonator is small in comparison with the shock formation distance, frequency $\omega$ of driving force differs slightly from the resonant frequency and the Q-factor is large, Q $>>1$ - the general equation (1) can be reduced [1] to the inhomogeneous Burgers equation:

$$
\frac{\partial U}{\partial T}+\Delta \frac{\partial U}{\partial \xi}-\pi \varepsilon \frac{\partial U}{\partial \xi}-D \frac{\partial^{2} U}{\partial \xi^{2}}=\frac{M}{2} \sin (\omega t)
$$

The following dimensionless variables and coefficients are used here:

$$
U=\frac{F}{c}, M=\frac{A}{c}, \xi=\omega t+\pi, T=\omega t / \pi, D=\frac{b \omega^{2} L}{2 c^{3} \rho}<1 .
$$

The first temporal variable $\xi$ describes "fast" oscillations, whereas the second one $T$ is responsible for "slow" evolution due to nonsteady-state build-up and decay processes, as well as for nonlinear and dissipative distortion of the wave profile. Parameter $D$ is the small ratio of the length of resonator to the characteristic absorption distance. The absorption coefficient $b$ and corresponding dissipative terms are not presented, for simplicity, in the initial functional equation (1), but this modifying can be easily performed in the differential equation (2). Exact analytical solutions to equation (2) for travelling waves were obtained in Refs. [2, 3]. These solutions can be adopted for calculation of standing nonlinear waves.

The scheme of solution consists of 2 stages. At first stage, the wave profile is calculated on the base of exact solutions to equation (2). This procedure and corresponding results are described in [4] not only for harmonic excitation, but for arbitrary periodic right-hand-side of equations (1) and (2). At the second stage, the wave field $u(x, t)$ is used for calculation of Q-factor and frequency response

\section{RESPONSE AND Q FOR QUADRATIC RESONATOR}

The nonlinear frequency response defined as rms velocity is shown in Fig.1. Curves are constructed for different amplitudes of vibration of the wall, corresponding to values $(M / \pi \varepsilon)=(1,4,9,16,25) \cdot 10^{-2}$. Along horizontal axis the dimensional discrepancy is shown, $\Delta / \pi \varepsilon ; \Delta=\pi\left(\omega-\omega_{0}\right) / \omega_{0}$, where $\omega_{0}$ is the resonant frequency. It is necessary to emphasize, that frequency response curves shown in Fig.1 are symmetric, their form is the same at positive and negative discrepancies.

Another definition as positive peak velocity of the wave profile is illustrated in Fig.2. The amplitude of vibration of the wall equals here to $(M / \pi \varepsilon)=0.09,0.25$ for two constructed curves. As distinct from Fig.1, these curves are not symmetric, because 
positive and negative half-periods of the wave profile are different; at positive discrepancies the positive peak of profile is higher than the negative one.
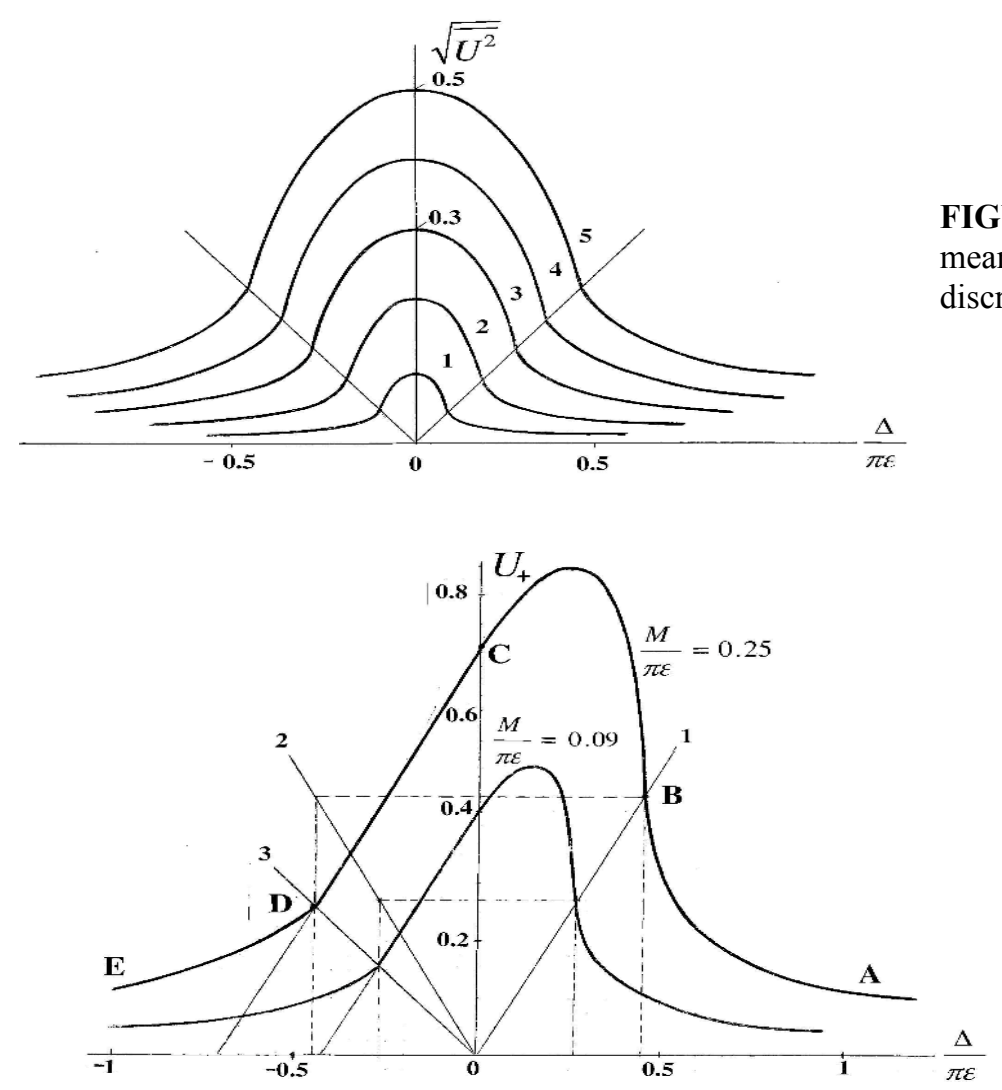

FIGURE 1. Quadratic resonator rootmean-square velocity as function of discrepancy parameter.
FIGURE 2. Quadratic resonator positive peak velocity as function of discrepancy parameter.

If linear dissipation caused by viscosity and thermal conductivity is taken into account, the exact expression for Q-factor is

$$
Q=\frac{1}{\sqrt{\pi \varepsilon M}}\left[-\frac{D^{2}}{\pi \varepsilon M} a_{0}\left(\frac{\pi \varepsilon M}{2 D^{2}}\right)\right]^{1 / 2},
$$

where $a_{0}(q)$ is the eigenvalue of the Mathieu function $\quad c e_{0}\left(\frac{\xi}{2}, \frac{\pi \varepsilon M}{2 D^{2}}\right)$. Numerical evaluation of nonlinear Q-factor for gaseous media at the wall vibration amplitudes $A \sim 10 \mathrm{~cm} / \mathrm{s}$ gives for $\mathrm{Q}$ the order of several tenths, whereas the corresponding linear $\mathrm{Q}$-factor for frequencies of order of $\mathrm{kHz}$ is 2-3 orders higher.

\section{CUBIC RESONATOR}

In acoustics cubically nonlinear systems are studied much less than quadratic. It is interesting to analyse the nonlinear behaviour of standing waves in cubic resonators because of two reasons. First of all, the physical evolution is radically different from the evolution of quadratic nonlinear waves. Secondly, cubic media are of great interest in connection with new applied problems. Such problems are connected with strong shear wave excitation for medical purposes [5-7], and with diverse geo-physical applications [8]. The approach described above can be successively applied to 
standing waves in cubic resonators, but now the idea of complete independence of counter-propagating waves breaks down. This method must be radically modified. The functional equation corresponding to the equation (1) now has the following structure

$$
F\left[\omega t+k L-\frac{\varepsilon}{c^{2}} k L\left(I+F^{2}\right)\right]-F\left[\omega t-k L+\frac{\varepsilon}{c^{2}} k L\left(I+F^{2}\right)\right]=A \sin (\omega t)
$$

where $I$ is the unknown integral of the squared wave profile. Consequently, the equation (5) is integro-functional. Nevertheless, it can be studied analytically. In some special cases it is reducible to cubic Burgers-type equation studied in Ref. [9] for plane waves and in Ref.[5, 10] for spatially-limited beams. Study of it is in process, however, some important results are already derived, and one is shown in Fig.3.

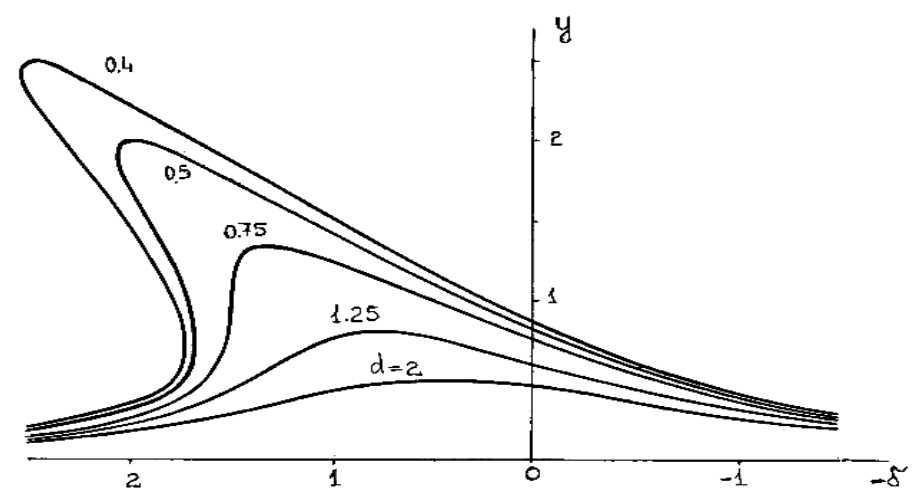

FIGURE 3. The cubic resonator frequency response as a function of discrepancy parameter.

That is the frequency response $y(\delta)$ for cubic resonator constructed for the following magnitudes of parameter $d=2,1.25,0.75,0.5,0.4$ (curves 1-5).

$$
\delta=\Delta\left(\frac{16}{3 \pi \varepsilon M^{2}}\right)^{1 / 3}, d=D\left(\frac{16}{3 \pi \varepsilon M^{2}}\right)^{1 / 3}, y=\frac{I}{c^{2}}\left(\frac{3 \sqrt{2} \pi \varepsilon}{M}\right)^{2 / 3}
$$

With absorption decrease the frequency response distorts. At weak absorption $d<\frac{\sqrt{3}}{2}$ the curve becomes non-single-valued at some positive values of discrepancy $\delta$.

\section{REFERENCES}

1. O.V.Rudenko, C.M.Hedberg and B.O.Enflo, Acoustical Physics 47, 452-460 (2001).

2. O.V.Rudenko, JETP letters 20, 203-204 (1974).

3. A.A.Karabutov, E.A.Lapshin and O.V.Rudenko, Sov.Phys.JETP 44, 58-63 (1976).

4. B.O.Enflo, C.M.Hedberg and O.V.Rudenko, J.Acoust.Soc.Am. 117(2), 601-612 (2004).

5. O.V.Rudenko and O.A.Sapozhnikov, Sov.Phys.JETP 79(2),.220-229 (1994).

6. A.P.Sarvazyan, O.V.Rudenko, S.D.Swanson, J.B.Fowlkes and S.Y.Emelianov. Ultrasound in Medicine and Biology .24,1419-1436 (1998).

7. S.Catheline, J.-L.Gennisson and M.Fink. J.Acoust.Soc.Am. 114, 3087-3091 (2003).

8. L.A.Ostrovsky and P.A.Johnson. La Rivista del Nuovo Cimento 24 (7), 1-47 (2001).

9. I.P.Lee-Bapty and D.G.Crighton, Philos.Trans.Royal Soc.London A 323, 173-209 (1987).

10. O.V.Rudenko and O.A.Sapozhnikov, Physics-Uspekhi 174(9), 973-997, (2004). 